\title{
Principios Sustentables y Parámetros Legislativos en una mina subterránea en México. Caso de Estudio
}

\section{Sustainable Principles and Legislative Parameters in an underground mine in Mexico. Case study}

MARTINEZ-TORRES, Rosa Eliał*, BEDNAREK, Mariusz, RIVERA-ACOSTA, Patricia y OJEDAGUTIERREZ, Maricela

Instituto Tecnológico de San Luis Potosí, Tecnológico Nacional de México

Universidad de Lodz, Polonia, Facultad de Ciencias Sociales

Universidad Politécnica de San Luis Potosí

ID $1^{\mathrm{er}}$ Autor: Rosa Elia, Martinez-Torres / ORC ID: 0000-0001-8936-9207, Researcher ID Thomson: X-3124-2018, CVU CONACYT ID: 953355

ID $1^{\text {er }}$ Coautor: Mariusz, Bednarek / ORC ID: 0000-0002-8402-4370

ID $2^{\text {do }}$ Coautor: Patricia, Rivera-Acosta / ORC ID: 0000-0002-8254-0005, CVU CONACYT ID: 232611

ID $3^{\text {er }}$ Coautor: Maricela, Ojeda-Gutierrez / ORC ID: 0000-0002-4655-2391, Researcher ID Thomson S-7636-2018, CVU CONACYT ID 740613

DOI: $10.35429 / J U S D .2019 .15 .5 .31 .34$

Recibido: 30 de Junio, 2019; Aceptado 30 Septiembre, 2019

\begin{abstract}
Resumen
La Organización de las Naciones Unidas, se postula a favor del Desarrollo Sostenible por medio de Objetivos, de los cuales, cuatro son estudiados a favor del medio ambiente; por su parte, el Marco Legislativo de México, provee a organismos de la Secretaría de Economía, facultad sobre parámetros ambientales que deben cumplir para que operen empresas del sector minero-metalúrgico. Con una metodología de estudio de caso, que forma parte de un caso múltiple integrado (Yin, 2013), se indaga acerca de las prácticas medio ambientales de una unidad de análisis (mina subterránea de mineral metálico en México), obteniendo información al aplicar instrumentos diseñados exprofeso y describir con un enfoque cualitativo la participación de los cuatro principios sustentables que obedecen a los Objetivos de Desarrollo Sostenible: (1) Industria, Innovación e infraestructura, (2) Ciudades y comunidades sostenibles, (3) Producción y consumo responsable y, (4) Vida y ecosistemas terrestres. El objetivo por consiguiente es: Articular el cumplimento de principios de sustentabilidad con los parámetros legislativos de una mina subterránea de mineral metálico, para contribuir a una propuesta integral de mejores prácticas para la Gestión Ambiental del sector minero-metalúrgico de México.
\end{abstract}

Sustentabilidad, Gestión Ambiental, Desarrollo Sostenible, Proceso minero-metalúrgico

\begin{abstract}
The United Nations Organization is in favor of Sustainable Development through Objectives, of which four are studied for their relationship with the environment; for its part, the Legislative Framework of Mexico, provides to the agencies of the Ministry of Economy, power over the environmental parameters that must be met for companies operating in the mining-metallurgical sector. With a case study methodology, which is part of an integrated multiple case study (Yin, 2013), the environmental practices of an analysis unit (underground metal ore mine in Mexico) were investigated, obtaining information on instruments designed and, describing with a qualitative approach, the participation of the four sustainable principles selected, which obey the Sustainable Development Goals: (1) Industry, innovation and infrastructure, (2) Sustainable cities and communities, (3) Production and consumption responsible and, (4) Life and terrestrial ecosystems Therefore, the objective is: Articulate compliance with the principles of sustainability with the legislative parameters of an underground metal ore mine, to contribute to a comprehensive proposal of best practices for the Environmental Management of the miningmetallurgical sector of Mexico.
\end{abstract}

Sustainability, Environmental Management, Mining and Metallurgical Process

Citación: MARTINEZ-TORRES, Rosa Elia, BEDNAREK, Mariusz, RIVERA-ACOSTA, Patricia y OJEDAGUTIERREZ, Maricela. Principios Sustentables y Parámetros Legislativos en una mina subterránea en México. Caso de Estudio. Revista del Desarrollo Urbano y Sustentable. 2019. 5-15: 31-40

\footnotetext{
* Correspondencia del Autor (m.rosaelia@gmail.com)

$\dagger$ Investigador contribuyendo como primer Autor
} 


\section{Introducción}

La Organización de las Naciones Unidas, realiza esfuerzos conjuntos con los gobiernos de los países para favorecer aspectos sociales, económicos y ambientales. Como resultado de este esfuerzo, nace la Agenda 2030, la cual alberga los Objetivos de Desarrollo Sostenible, que deberán ser conseguidos de forma equilibrada en razón del crecimiento. En México, estos esfuerzos son equivalentes a aquellos apegados a Ley. En el sector minerometalúrgico, estas obligaciones se hacen cumplir y son evaluadas por organismos exclusivos bajo la ejecución de la Secretaría de Medio Ambiente y Recursos Naturales y la Procuraduría Federal de Protección al Ambiente; dichas obligaciones se describen en el documento oficial "Manifiesto de Impacto Ambiental", parámetros que se han definido como fundamentales en las evaluaciones para su operatividad.

La Industria Minero-metalúrgica está considerada como un sector productivo que mantiene factores de crecimiento económico estables, sin embargo, por la naturaleza de su proceso industrial, pone en riesgo el entorno, tanto en emisiones hacia aire, suelo y agua, como la devastación de recurso natural.

Ante la importancia de esta industria, se debe prestar atención en que su operatividad, se concentre en minimizar o de ser posible erradicar los impactos ambientales, sin sacrificar productividad y rentabilidad. Con base en los esfuerzos, tanto internacional como nacional, para mentener el equilibrio económico, social y ambiental, se gesta una investigación dentro del sector minerometalúrgico, en donde se obtienen propuestas para mejorar las prácticas que las empresas del sector orientan hacia el cuidado del medio ambiente.

Con un enfoque cualitativo y paradigma constructivista del tipo descriptivo, se diseña un estudio de caso múltiple integrado (Yin, 2013), método que permite tomar empresas del sector minero-metalúrgico como unidades de análisis. Dichas unidades seleccionadas por criterios de conveniencia, cumplen diferentes aspectos dentro del proceso industrial, logrando ser una muestra representativa (Hernández, 2014). La metodología que se sigue se establece con análisis teóricos fundamentados en sustentabilidad.
El estudio de Principios Universales de Sustentabilidad, extraídos de los Objetivos de Desarrollo Sostenible, establecen el eje motor de la investigación integral; los parámetros legislativos, se evalúan a través de acciones generadoras de impactos o riesgos de impacto ambiental para corroborar su efectividad.

La triangulación de estos elementos: fundamento teórico, principios sustentables y, parámetros legislativos, promueven el diseño de un modelo sustentable para la Industria Minerometalúrgica, por lo que se lleva a cada unidad de análisis para lograr una validación; de esta manera, la investigación se conforma de casos de estudio individuales, siguiendo el método de forma sistemática.

En el presente documento, se encuentra un fragmento de esta investigación integral, que tiene un objetivo parcial de describir por medio de la articulación de los elementos mencionados, la realidad de las prácticas medio ambientales en una unidad de análisis, para de ser posible, una vez que se implemente el modelo sustentable y se tenga una guía de mejores prácticas, este esfuerzo forme parte de la Gestión Ambiental de las empresas del sector. Dicha unidad, cumple criterios de selección de tipo de mineral (metálico), tipo de explotación (mina subterránea) y su proceso productivo se encuentra en operación.

\section{Bases Teóricas}

\section{Gestión ambiental}

Massolo (2015), ha descrito la Gestión Ambiental como el conjunto de acciones y estrategias mediante las cuales se organizan actividades destinadas para influir sobre el ambiente, logrando como resultado, calidad de vida, advertencias de problemas al entorno, partiendo conceptualmente del desarrollo sostenible, por lo que tiene como finalidad, conseguir el equilibrio adecuado para el crecimiento económico y poblacional, el uso racional de los recursos y la protección y conservación del medio ambiente.

Es la guía o método de trabajo para conseguir conductas específicas que estén alineadas con metas, objetivos y normas, a favor de la minimización de riesgos ambientales, fomentando precisiones sociales, financieras, económicas y competitivas (Massolo, 2015).

MARTINEZ-TORRES, Rosa Elia, BEDNAREK, Mariusz, RIVERAACOSTA, Patricia y OJEDA-GUTIERREZ, Maricela. Principios Sustentables y Parámetros Legislativos en una mina subterránea en México. Caso de Estudio. Revista del Desarrollo Urbano y Sustentable. 2019 
El Programa de Naciones Unidas para el Medio Ambiente, PNUMA (2010), define gestión ambiental como la mejora del ambiente a través de los procesos productivos, adhiriendo a ellos, sistemáticamente, el concepto de desarrollo sustentable y la mitigación de problemas ambientales, lo que permite calidad de vida y bienestar económico-social, de esta manera, puede considerarse como un mecanismo para conseguir desarrollo económico y humano con el uso racional de recursos naturales y protección del medio ambiente.

Ante estas dos posturas, la importancia de definir Gestión ambiental para este documento, radica en la finalidad que tiene, al considerar ámbitos como el humano o social, la economía o rentabilidad y los aspectos ambientales como mitigación o bien minimización de riesgos, todos ellos, encauzados por el desarrollo sostenible.

\section{Desarrollo Sostenible}

El Desarrollo Sostenible propone un crecimiento económico que implica: cohesión social, naturaleza limitante de los recursos naturales y capacidad ambiental para asimilar los residuos (ONU, 1984). Promueve además, el equilibrio entre el crecimiento económico, la justicia social y el medio ambiente; la base es la prevención financiera para los elementos de remediación, la inclusión de hábitos humanos que tienden a la equidad del consumismo y la creación de servicios y oportunidades, y procesos de producción y operación más eficientes (Naredo, 1996).

Emanan como resultado de esfuerzos globales en torno al desarrollo sostenible, 17 Objetivos, descritos en la "Agenda 2030", logrando con ellos, que economías importantes, se unan para enfrentar los desafíos económicos, sociales y ambientales (Martínez y Rivera, 2017). Cabe destacar la parte operativa que el Desarrollo Sostenible pronuncia: se fundamenta en los aspectos o procesos productivos $\mathrm{y}$ además, lleva intrínseco la estructura de cómo se logre, por ello se hace énfasis además, en los Objetivos de Desarrollo Sostenible, de los cuales se consideran cuatro para medirlos en la industria minero-metalúrgica en este estudio.

\section{Sustentabilidad}

Naredo (1996) afirma que la Sustentabilidad se refiere al crecimiento regulado, involucrando medidas políticas y sociales, para utilizar los recursos naturales de manera eficiente; es un medio para satisfacer las necesidades actuales del ser humano, sin comprometer los recursos (Naredo, 1996). La Sustentabilidad forma parte de la planificación estratégica, centrándose en los impactos ecológicos y espaciales, siendo este último (1) el suministro (consumo de agua, energía, combustibles) y (2) el saneamiento (residuos sólidos, aguas residuales, gases tóxicos); promueve visualizar los problemas con fundamentos científicos y éticos, calcula límites mínimos y máximos que mantengan el nivel de vida y el desarrollo económico. (Naredo, 1996).

Para fines de este estudio, Desarrollo Sostenible es la parte operativa, son actividades, metas, es decir, propuestas tangibles; por su parte, la Sustentabilidad, se remite a la generación de políticas y estrategias para perseguir la operatividad del desarrollo sostenible. De esta manera, se obtiene una triangulación importante, ya que la gestión ambiental, cobija en ese esfuerzo a la sustentabilidad, por medio de la Planeación Estratégica.

\section{Proceso Minero-metalúrgico}

La Coordinación general de Minería (2014) describe las etapas del proceso minerometalúrgico:

- Exploración: se reconocen las áreas de interés, a fin de confirmar las características físicas y químicas que muestran que los depósitos minerales existen.

- Explotación: trabajos diseñados para preparar y desarrollar el área donde hay depósitos minerales, así como actividades dirigidas a extraer dichos minerales o sustancias de la naturaleza.

- Beneficio de los minerales (metalurgia): trabajos de preparación y tratamiento, fundición, concentración y refinación de productos minerales extraídos, con el fin de recuperar minerales y sustancias en términos de pureza (separación). 
Cierre: una vez que finaliza la operación minero-metalúrgica, el cierre debe planificarse estratégicamente, con la visión de reintegrar la propiedad utilizada a su entorno natural o urbano

El estudio se desarrolla a partir del conocimiento de las actividades propias de cada etapa de este proceso industrial.

\section{Bases Metodológicas}

El presente trabajo, forma parte de una investigación integral que profundiza en el desempeño de las acciones ambientales de una muestra representativa que la conforman cuatro unidades que pertenecen al sector minerometalúrgico de México. La metodología empleada para perseguir el objetivo de la investigación es mediante un caso de estudio múltiple integrado (Yin, 2013), lo que permite a los investigadores, realizar cuatro casos de estudio en unidades diferentes, bajo las mismas condiciones de método, de donde extrae información por medio de instrumentos diseñados ad hoc y, obedeciendo a la técnica de observación participativa (Hernández, 2014).

Las unidades de análisis, pertenecen a empresas representativas del sector minero metalúrgico que cumplen con criterios fundamentales. De forma general se dividen en (1) minas, con características propias de sistemas de explotación (mina subterránea y mina a cielo abierto) y localización geográfica $\mathrm{y}$, (2) refinerías, empresas del sector que continúan el proceso metalúrgico una vez que sale el mineral concentrado de las minas (Martínez y Rivera, 2017).

La selección se hace por conveniencia (Hernández, 2014) tomando criterios como: localización, permisibilidad, sistema de explotación, tipo de unidad. Se enlistan a continuación el total de las unidades seleccionadas:

\begin{tabular}{|l|l|l|}
\hline Unidad Seleccionada & \multicolumn{1}{c|}{ Unidad } & \multicolumn{1}{c|}{ Mineral extraído o concentrado } \\
Mina subterránea & $\begin{array}{l}\text { MSJ } \\
\text { Capital canadiense }\end{array}$ & $\begin{array}{l}\text { Metálico } \\
\text { Mina a cielo abierto }\end{array}$ \\
\hline Refinería & $\begin{array}{l}\text { JRU } \\
\text { Pequeño minero }\end{array}$ & $\begin{array}{l}\text { Concentrado de fluorita - } \\
\text { No metálico }\end{array}$ \\
\hline Unidad en cierre & $\begin{array}{l}\text { REZ } \\
\text { Grupo mexicano }\end{array}$ & $\begin{array}{l}\text { Zinc, Ácido Sulfúrico } \\
\text { Metalurgia }\end{array}$ \\
& $\begin{array}{l}\text { MSX } \\
\text { Capital extranjero }\end{array}$ & Oro (Doré), plata \\
\hline
\end{tabular}

Tabla 1 Selección de unidades de análisis por criterios de conveniencia

Fuente: Elaboración Propia
En la tabla 1, puede observarse la mina subterránea, que es la que en el presente documento, se describe de forma individual como unidad de análisis, bajo el método de caso de estudio, con un enfoque cualitativo y un paradigma constructivista del tipo descriptivo.

\section{Caso de estudio mina subterránea}

\section{Descripción del Problema}

Los efectos negativos del desarrollo industrial minero-metalúrgico se observan en la ecología $\mathrm{y}$ en las sociedades a las que su impacto afecta (Martínez y Bednarek, 2018). Los problemas del tipo ambiental, debido a las malas prácticas que se han realizado dentro del sector minero, son consecuencia de la inexistencia o falta de aplicación de lineamientos de regulación y legislación a través del tiempo, ya que se han identificado sitios contaminados con compuestos orgánicos e inorgánicos derivados de los procesos de explotación, extracción y el beneficio de minerales. (Volke y Velasco, 2002).

\section{Justificación}

La relevancia de realizar este estudio, se basa en la descripción del tratamiento de los tópicos medio ambientales internacionales, focalizando este estudio en principios de Sustentabilidad.

La originalidad radica en la conexión de tópicos medio ambientales internacionales con los parámetros que la Ley Minera de México exige, para poder operar.

\section{Objetivo}

El objetivo general que se persigue es: articular el cumplimento de principios de sustentabilidad con los parámetros legislativos de una mina subterránea de mineral metálico, para posteriormente, contribuir a una propuesta integral de mejores prácticas para la Gestión Ambiental en el sector minero-metalúrgico de México.

\section{Objetivos específicos}

1. Realizar la conexión entre los parámetros de ley y los principios sustentables que en la actualidad, la unidad de análisis presenta. 
2. Describir las observaciones de cada principio sustentable en las etapas de su proceso productivo, como expresión directa de las prácticas medioambientales actuales.

\section{Desarrollo}

Se implementó una metodología sistemática para documentar el caso de estudio desde la solicitud hacia la Gerencia, hasta la culminación de la integración de todas las unidades; se hizo énfasis en que era indispensable que una persona de la unidad, con autoridad suficiente, acompañara a los investigadores en el trabajo de campo, que incluía visitas a la unidad para reconocimiento y la aplicación de instrumentos de recolección de datos: guías de observables, listas de verificación (de datos no observables) y entrevistas del tipo complementario de información, los cuales fueron diseñados exclusivamente para la investigación y avalados y validados por expertos en minería ambiental.

\section{Principios sustentables a valorarse}

Estos son extraídos, luego de un análisis concienzudo a partir de los 17 Objetivos de Desarrollo Sostenible (ONU, 2010) que se encuentran descritos como parte de las metas que se persiguen en la "Agenda 2030":

Industria, innovación e infraestructura: Invertir en infraestructura, empodera a las comunidades, logra incrementos de productividad, ingresos y mejoras en resultados sanitarios y educativos. El crecimiento y urbanización genera necesidad de contar con nuevas inversiones en infraestructuras sostenibles que permiten que las ciudades sean más resistentes al cambio climático e impulsan crecimiento económico y estabilidad social.

Ciudades y comunidades sostenibles: Las ciudades han permitido que las personas progresen social y económicamente. Los problemas de las ciudades son congestión, falta de fondos para servicios básicos, escasez de vivienda y deterioro de infraestructura, por lo que su reto es enfrentarlos para prosperar y crecer aprovechando mejor los recursos y reduciendo contaminación. Se requieren ciudades con oportunidades, acceso a servicios, energía, vivienda, transporte y más facilidades.
Las ciudades se caracterizan por ser centros que concentran comercio, cultura, ciencia, productividad, creatividad, desarrollo social y económico.

Producción y consumo responsable: Fomentar uso eficiente de recursos y eficiencia energética, infraestructuras sostenibles y facilitar acceso a servicios básicos, empleos ecológicos y decentes, mejora calidad de vida. Aplicarlo ayuda a lograr planes de desarrollo, reduce costos económicos, ambientales y sociales, aumenta la competitividad. Crear más y mejores cosas con menos recursos, incrementando ganancias netas mediante la reducción de utilización de recursos, degradación y contaminación, logrando mejor calidad de vida. Adopción de enfoque sistémico para lograr cooperación entre participantes: empresas, consumidores, gobierno, organismos, investigadores, científicos.

Vida de ecosistemas terrestres: Promover el uso sostenible de ecosistemas terrestres, actuar contra la desertificación, intentar detener e invertir la degradación de las tierras y frenar la pérdida de la diversidad biológica. La naturaleza, vista como ecosistemas, ocupa el $30 \%$ de la superficie de la tierra, lo que proporciona seguridad alimentaria y refugio, fundamentales para combatir el cambio climático, al proteger la diversidad biológica. Los retos para el desarrollo sostenible son la deforestación y desertificación humana, mediante restablecimiento de ecosistemas.

\section{Parámetros legislativos}

Se describen 109 parámetros dentro del documento oficial "Manifiesto de impacto Ambiental" que obliga la Ley Minera en el país, a dar puntual cumplimiento por parte de las empresas del sector. De entre estos, 15 son, después de análisis literarios y preparación metodológica de investigación (Mariusz y Bednarek, 2018), considerados para medir y evaluar en los instrumentos de recogida de datos diseñados; la interpretación que de ellos se genera, se realiza mediante la conexión de éstos con los principios de sustentabilidad. Los parámetros son: 
Tiempo de vida útil, responsable técnico, Naturaleza del proyecto, dimensiones, uso de suelo, urbanización del área, preparación del sitio, construcción de obras, operación y mantenimiento, abandono, uso de explosivos, generación y manejo de residuos, infraestructura para el manejo y disposición de residuos, aspectos abióticos, aspectos bióticos $\mathrm{y}$, paisaje.

\section{Contextualización de la Unidad de Análisis}

La mina subterránea, unidad de análisis de este estudio de caso, se encuentra localizada en el estado de Zacatecas, pertenece a una cabecera municipal que tiene una población de 21,398 habitantes, las concesiones mineras ascienden a 1,500 hectáreas, que han sido explotadas desde 1760 con pequeños trabajos de mina $^{1}$ bajo la supervisión de hacendarios. Los permisos de operación de actividades mineras, o fundos mineros, pertenecieron a personas físicas hasta 1890, cuando los adquiere grupos mineros mexicanos fortalecidos entre 1948 y 1995, que intervienen en exploración con tecnología avanzada y además invierten en obra minera, surgiendo problemas de los denominados "grupos radicales" encabezados por campesinos de la zona, que orillan al grupo a abandonar la operación. Re-inicia actividades en 1995 (Martínez, 2000)

Desde 2016 la unidad es explotada y beneficiada por un corporativo con capital canadiense, quienes además montaron infraestructura adecuada para la extracción, la cual proviene de otras minas cercanas. El traslado de esta infraestructura fue simultáneo a la exploración, lo que generó una expansión territorial potencial de explotación. Se trata de una mina subterránea, localizada en un Trend ${ }^{2}$ metálico, mantiene un sistema de explotación en subniveles con barrenación larga, aprovechando las características de la potencia del yacimiento. La extracción se realiza con camión convencional de 8 y $12 \mathrm{mt}^{3}$, lo que sugiere trayectos espaciosos dentro de la mina; su sistema de beneficio es el de flotación, generando deshechos que son arrojados en una presa de jales ubicada en tres costados del cerro contiguo a la unidad.

\footnotetext{
${ }^{1}$ Se refiere a actividades con métodos precarios o sin proceso formal de extracción de mineral; usado en los orígenes de la minería.

2 Trend: alineación de estructuras metálicas como franjas verticales que cruzan la república mexicana.

ISSN 2414-4932

ECORFAN® Todos los derechos reservados
}

El personal asciende a 200 empleados, entre contratos directos e indirectos. La producción está proyectada para el año 2021.

\section{Resultados}

Se presentan los resultados del caso de estudio de la unidad de análisis, mina subterránea, que para efectos de la investigación integral, se les considera preliminares. Como resultado, se obtiene, luego del trabajo de campo, la articulación de dos elementos (principios sustentables y parámetros legislativos) bajo el fundamento teórico descrito de sustentabilidad adherido a Gestión Ambiental.

Para llegar al resultado deseado, previamente se realizó una primera relación del proceso productivo (por etapas como primera fase, después global para fines de presentación) frente a los principios, obedeciendo a las metas por estos perseguidas. Esta relación se realiza considerando como efecto dependiente, los impactos y riesgos de impactos ambientales derivados de actividades del proceso industrial.

\begin{tabular}{|c|c|}
\hline $\begin{array}{l}\text { Principio } \\
\text { Sustentable }\end{array}$ & $\begin{array}{l}\text { Acciones que ocasionan impactos o riesgos de } \\
\text { impacto ambiental }\end{array}$ \\
\hline $\begin{array}{l}\text { Industria, } \\
\text { innovación } \\
\text { infraestructura }\end{array}$ & $\begin{array}{l}\text { Construcción de caminos para maquinaria de } \\
\text { exploración. } \\
\text { Construcción de Plazas. } \\
\text { Mantenimiento de equipo en campo, uso de } \\
\text { suministros. } \\
\text { Desplazamiento de material en cielo abierto y en } \\
\text { desarrollos subterráneos (terreros y tepetateras). } \\
\text { Construcción de talleres para servicios. } \\
\text { Obtención de jales como desecho. } \\
\text { Uso y contención de reactivos químicos. } \\
\text { Uso en exceso y contaminación de agua. } \\
\text { Derrames. } \\
10 \text { Estudios técnicos y supervisiones incompletas. } \\
11 \text { Normas no cumplidas. }\end{array}$ \\
\hline $\begin{array}{l}\text { Ciudades } \\
\text { comunidades }\end{array}$ & $\begin{array}{l}\text { Construcción de caminos y pasos sin considerar } \\
\text { poblaciones. } \\
\text { Creación de Organizaciones No Gubernamentales } \\
\text { (ONG). } \\
\text { Uso de explosivos sin estudios técnicos previos } \\
\text { Recursos económicos limitados - no apoyo a la } \\
\text { comunidad. }\end{array}$ \\
\hline $\begin{array}{l}\text { Producción } \\
\text { consumo } \\
\text { responsables }\end{array}$ & $\begin{array}{l}\text { Desplazamiento de material por obra de exploración. } \\
\text { Tocar nivel freático. } \\
\text { Construcción de lumbreras. } \\
\text { Construcción de tiros de mina subterránea. } \\
\text { Construcción de socavones. } \\
\text { Extracción de gases a la atmósfera. } \\
\text { Consumo excesivo y contaminación de agua. } \\
\text { Almacenamiento de reactivos obsoletos en planta de } \\
\text { beneficio y laboratorios. } \\
\text { Construcción de presa de jales sin taludes. }\end{array}$ \\
\hline $\begin{array}{l}\text { Vida } \\
\text { ecosistemas } \\
\text { terrestres }\end{array}$ & $\begin{array}{l}\text { Construcción de caminos. } \\
\text { Construcción de plazas. } \\
\text { Uso de aceites. } \\
\text { Formación de terreros. } \\
\text { Formación de tepetateras. } \\
\text { Construcción de naves: infraestructura. } \\
\text { Obtención de jales como desecho. } \\
\text { Programación económica insuficiente o nula para } \\
\text { etapas de cierre y remediación. }\end{array}$ \\
\hline
\end{tabular}

Tabla 2 Acciones generadoras de Impactos o Riesgos de Impacto ambiental

Fuente: Elaboración Propia

MARTINEZ-TORRES, Rosa Elia, BEDNAREK, Mariusz, RIVERAACOSTA, Patricia y OJEDA-GUTIERREZ, Maricela. Principios Sustentables y Parámetros Legislativos en una mina subterránea en México. Caso de Estudio. Revista del Desarrollo Urbano y Sustentable. 2019 
Con base en esta información, se realiza la relación que éstas acciones tienen con los 15 parámetros legislativos, quedando de forma directa, relacionados los principios sustentables y los parámetros. La siguiente información es revisada y avalada por los expertos mineros ambientalistas, se presenta en la tabla $3^{\mathrm{a}}$ y $3 \mathrm{~b}$ a manera de resumen:

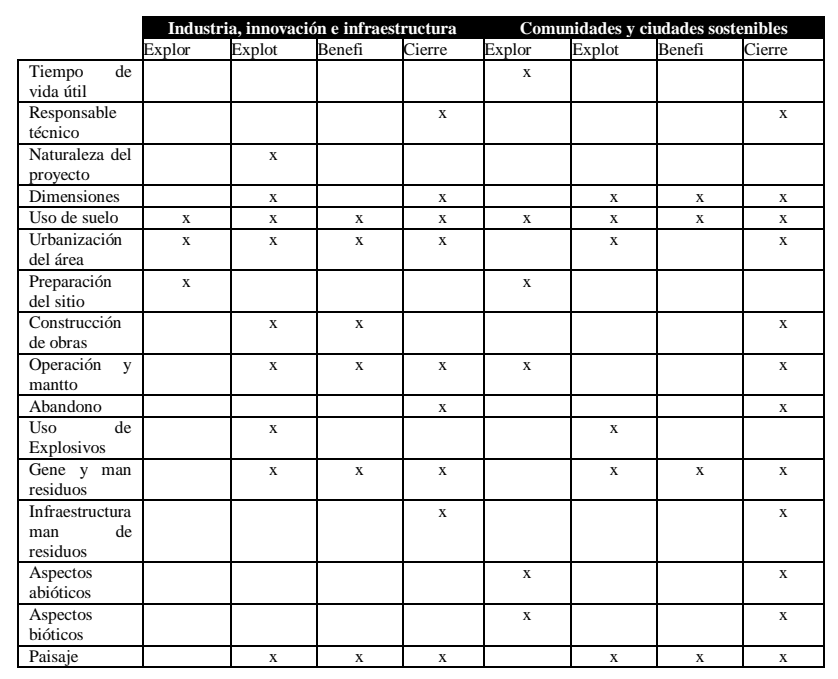

Tabla 3a Relación Principios Sustentables y Parámetros Legislativos

Fuente: Elaboración Propia

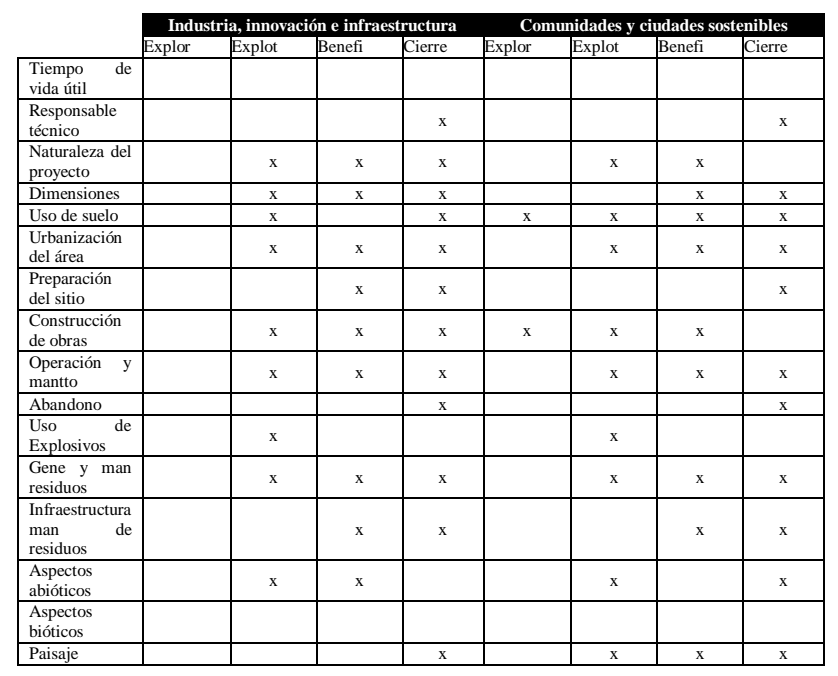

Tabla 3b Relación Principios Sustentables y Parámetros Legislativos

Fuente: Elaboración Propia

Como resultados alternativos, luego de obtener, analizar e interpretar la articulación entre Principios Sustentables y Parámetros Legislativos, a través de los posibles generadores de riesgos ambientales por etapa del proceso productivo, se tienen las descripciones sobre lo observado de cada Principio Sustentable en relación a la ubicación en ellos, de los parámetros.
Cabe mencionar, que ésta descripción, no es una interpretación o evaluación, solamente se resume lo observado de la relación que sustenta esta fracción de la investigación:

1. Industria, innovación e infraestructura: Actualmente no se realizan actividades de exploración en superficie, las planillas de barrenación aperturadas no se hicieron sobre terrenos forestales. Se depende de empresas subcontratadas a quienes se exige el mantenimiento del equipo en condiciones óptimas, en la unidad, cada taller cuenta con planchas de concreto para realizarlo y hay fosa de captación del residuo peligroso que es almacenado de forma temporal, conforme lo dicta el procedimiento que para esta actividad tienen. En la formación de terreros y tepetateras se ubicó en áreas no protegidas. Se tienen jales como desecho, éste es trasladado a la presa por medio de tubería de polietileno de alta densidad, su descarga se realiza mediante hidrociclones con acoplamientos termofusionados para evitar riesgos de fuga. El diseño de la presa, que es del tipo convencional, permite utilizar el material removido del vaso como material de préstamo para la elaboración de los bordos, lo que evita impactos adicionales de sitio. Los estudios de impacto ambiental están en orden según registros de entrega, seguimiento y evaluación de la autoridad correspondiente. En caso de impactos ambientales no medidos, se tiene un plan de emergencia avalado también por expertos y responsable técnico de la empresa. 


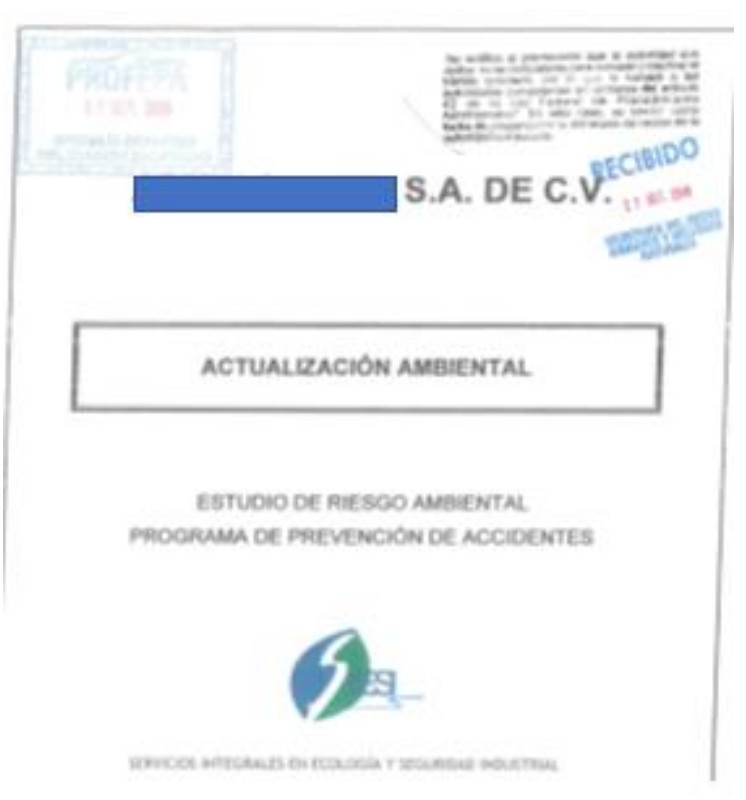

Figura 1 Evidencia de actualización de informes ambientales

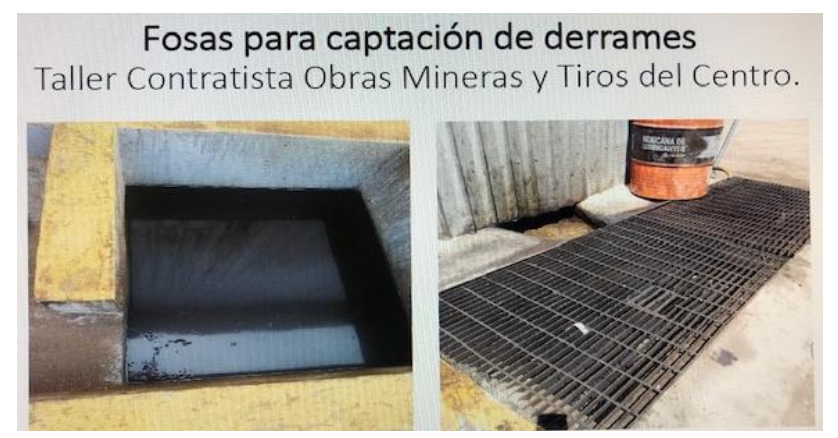

Figura 2 Evidencia de fosas de captación de deshecho industrial

A manera de evidencia, las figuras anteriores son mostradas, para sustentar la descripción brindada de lo observado en las visitas de campo a la unidad de análisis.

2. Ciudades y comunidades sostenibles: Históricamente, la unidad de análisis, ha sido sujeto de problemas sociales, encabezado por campesinos de la zona, demandando daños por detonaciones en algunos sitios del pueblo. Esto ha traído consecuencias operativas, una de ellas, la localización y diseño de polvorines, los cuales son evaluados con énfasis por parte de las autoridades. Básicamente, este rubro lo minimizan a programas de responsabilidad social por medio del Departamento de relaciones comunitarias, el cual, a partir de presupuesto programado, brinda apoyo a solicitudes de las localidades aledañas, expresando aun así, que existen diferencias entre lo solicitado y lo ofrecido.

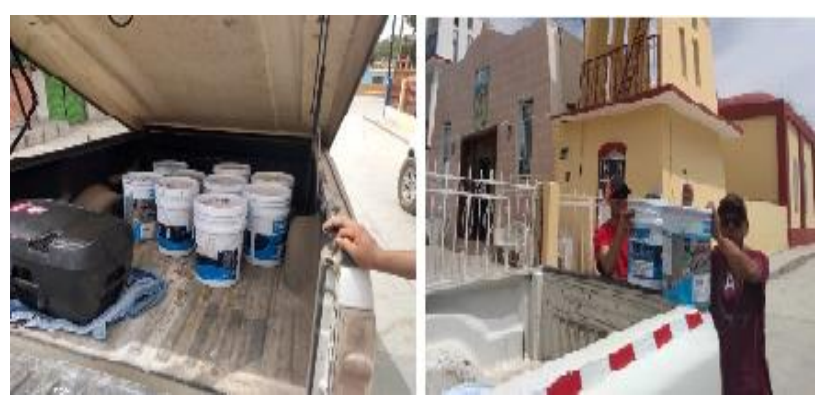

Figura 3 Apoyo de rehabilitación a Iglesia de comunidad aledaña

3. Producción y consumo responsable: $\mathrm{La}$ Productividad de la unidad se mide con base en la producción de mineral extraído: mineral mineralizado y materia estéril, en relación al suministro para ello utilizado. El material mineralizado es enviado a la planta de beneficio, mientras que el material estéril se envía a tepetateras. Al interior de la mina, se refuerza la ventilación, ya que atienden actualmente proyectos de diseño y ubicación de refugios de salvamento de personal y trabajo de obra de caminos o salidas de emergencia (rampas, contrapozos). El uso de agua y de neutralizadores de soluciones químicas en patios de lixiviación, planta de beneficio y aún en mina, son bastos; algunos problemas sociales se originan a partir de este dato, ya que han sido demandados por contaminación de mantos freáticos en años próximos pasados. Cuentan con un sistema de bombeo, en donde el agua utilizada al interior mina, es re-utilizada en superficie, puesto que no cuenta con indicadores fuera de límites permitidos de compuesto químico. En cuanto a la calidad de aire, no hay monitoreo de gases en sitios de instalación de extractores de interior mina, así como tampoco hay dosificadores automatizados de reactivos, realizada por ende, por los empleados de forma manual. Los desechos se transportan a la presa de jales con ayuda de hidrociclones. 


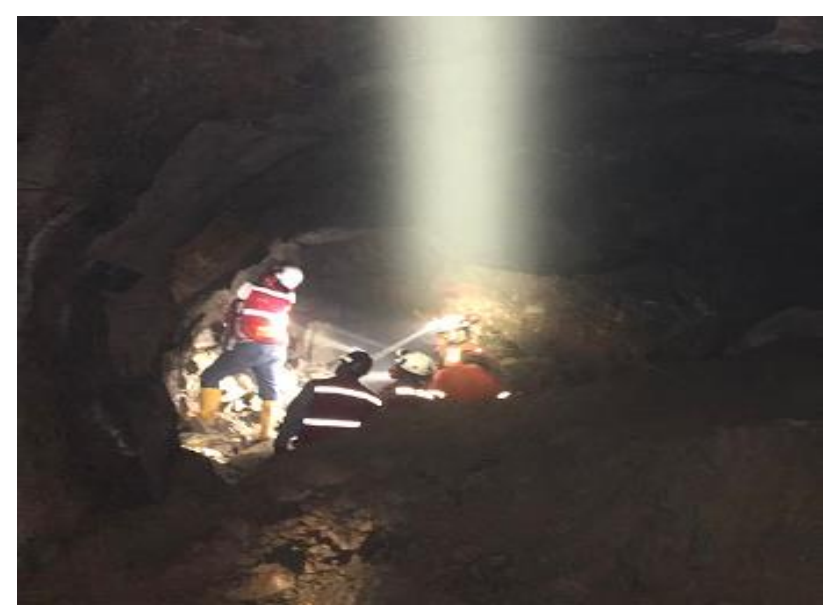

Figura 4 Cuadrilla valorando proyecto "Contrapozo". Interior mina

4. Vida de ecosistemas terrestres: Actualmente, la proyección de vida de la unidad, se extiende hasta el año 2021. $\mathrm{Su}$ proyecto de abandono, está planificado y descrito en el "Manifiesto de Impacto Ambiental" autorizado por los organismos pertinentes. Se contemplan temas como hidrología (superficial y subterránea), atmósfera (aire y ruido), suelo, flora y fauna, medio socioeconómicos y paisaje. El material estéril que forma tepetateras, será utilizado para relleno de obras en donde se extrajo mineral. El total de instalaciones se prevé sea desmantelado; además existen documentos que avalan estudios de devastación para que ésta sea medida en riesgos y remediada en su momento de cierre. Cuentan con una partida financiera para prevención y remediación de abandono de cada proyecto que se ha gestado a partir de la actual administración. El cierre de operaciones no se realizará a corto plazo, sin embargo se mantienen abiertos canales de comunicación con los pobladores acerca de tipos de minado (explosiones), cierres y progreso de las obras mineras (mina). El contorno de la presa de jales, está siendo reforestado, con referencia al plan de cierre, esta presa describe pobremente, cómo sería remediada.
Junio 2019 Vol.5 No.15 31-40

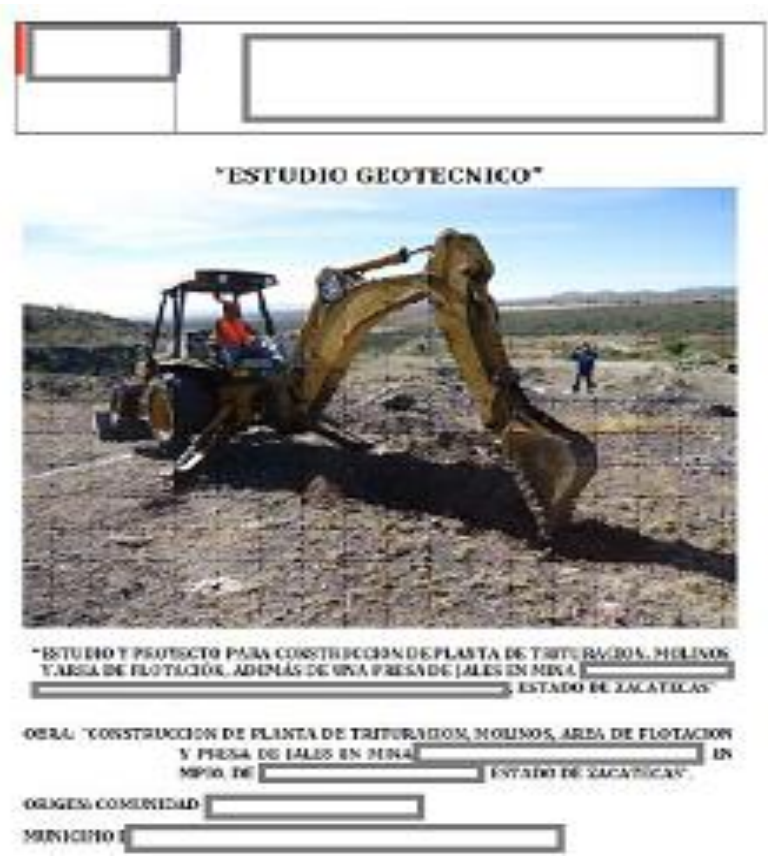

Figura 5 Evidencia de estudios existentes relacionados con devastes

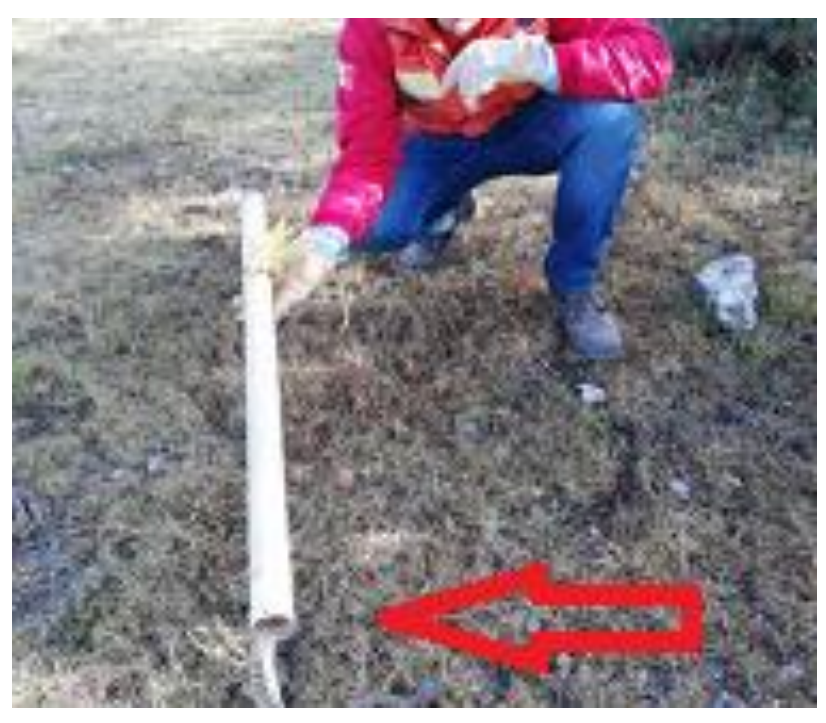

Figura 6 Evidencia de cuidados con especies de la zona.

\section{Conclusiones}

Esta unidad de análisis es representativa de la Industria Minero-metalúrgica, pues se trata de una mina subterránea, la típica mina, de mineral metálico, que tienen mayor reconocimiento por su uso y aplicación en la vida cotidiana, en este caso Plata. El trabajo de campo de este estudio de caso, fue determinante en la investigación, México es primer productor de plata a nivel mundial, lo que significa que esta unidad participa activamente en la contribución del metal a factores económicos. 
La unidad cuenta con capital canadiense, lo que la conduce a mantener sistemas altos de operatividad, ya que este tipo de inversionistas, normalmente cotiza en la bolsa de valores. Para México representa además, estabilidad en el sector al equilibrase la inversión contra lo exportado como mineral concentrado.

El caso de estudio contiene información representativa para la investigación global, la cual una vez que las cuatro unidades de análisis seleccionadas sean integradas e interpretadas en conjunto, además de significar que el modelo sustentable está validado, brindará la oportunidad por medio de una guía de mejores prácticas, de colaborar con la minimización de impactos y riesgos de impactos ambientales.

En forma particular, la percepción general de los investigadores, radica en las observaciones de cumplimiento de la normatividad, sin embargo, sí es compleja la interpretación conforme los principios sustentables, pues hay aún tendencias en el análisis. Ésta interpretación, aún está definiéndose en la forma conjunta, puesto que el modelo es flexible, pero con fundamentos explícitos, por lo que debe ajustarse. Cabe la anotación de que cada unidad de análisis tiene diferencias propias del proceso productivo en cada etapa, pues viven diferentes momentos en su operatividad.

El objetivo de este documento fue cumplido, el formato de cada estudio de caso arroja información importante que conducirá al cumplimiento del objetivo general de la investigación.

\section{Referencias}

Coordinación General de Minería, (2014). Guía para conocer las etapas del proceso productivo para la pequeña Minería. México: Secretaría de Economía.

Hernández, R., (2014). Metodología de la Investigación, 6ta edición. México: McGrawHill.

Massolo, L., (2015). Introducción a las herramientas de gestión ambiental. Argentina: Editorial de la Universidad de la Plata.
Naredo, J., (1996). Sobre el origen, el uso y el contendio del término sostenible. En la construcción de la ciudad sostenible. España: Ed. Ministerio de Obras Públicas, Transportes y Medio Ambiente.

Martínez, R., (2000). Informe Técnico La Perreña. Edición interna Peñoles.

Martínez, R.E. y Bednarek, M., (2018). Fundamentos para construcción de Instrumento Ambiental para la Industria Minerometalúrgica. Revista de Arquitectura y Diseño: ECORFAN-Spain.

Martínez R., and Rivera, P., (2017). Articulación de los objetivos de Desarrollo Sostenible y la Legislación Ambiental en la Industria Minera. REMINEO. 2017

Organización de las Naciones Unidas, (2010). Agenda 2030 para el Desarrollo Sostenible. ONU.

Volke, T., Velasco J., (2002). Tecnologías de remediación para suelos contaminados. México: INE-SEMARNAT.

Yin, R., (2013). Validez y generalización en futuras evaluaciones de casos de estudio. COSMOS corporation. 